\title{
MODIFICATION OF THE CURRENT PROFILE IN DIII-D BY OFF-AXIS ELECTRON CYCLOTRON CURRENT DRIVE
}

\author{
by \\ T.C. LUCE, Y.R. LIN-LIU, R.W. HARVEY, 1 \\ G. GIRUZZI, ${ }^{2}$ J.M. LOHR, C.C. PETTY, \\ P.A. POLITZER, R. PRATER, and B.W. RICE ${ }^{3}$
}

This is a preprint of an invited paper presented at the 26th European Conference on Controlled Fusion and Plasma Physics, June 14-18, 1999, Maastricht, The Netherlands, and to be printed in Plasma Physics and Controlled Fusion.

Work supported by

U.S. Department of Energy Contracts DE-AC03-99ER54463 and W-7405-ENG-48

1CompX

2CEA, Centre d'Études de Cadarache

${ }^{3}$ Lawrence Livermore National Laboratory

GENERAL ATOMICS PROJECT 30033

JULY 1999 


\section{DISCLAIMER}

This report was prepared as an account of work sponsored by an agency of the United States Government. Neither the United States Government nor any agency thereof, nor any of their employees, make any warranty, express or implied, or assumes any legal liability or responsibility for the accuracy, completeness, or usefulness of any information, apparatus, product, or process disclosed, or represents that its use would not infringe privately owned rights. Reference herein to any specific commercial product, process, or service by trade name, trademark, manufacturer, or otherwise does not necessarily constitute or imply its endorsement, recommendation, or favoring by the United States Government or any agency thereof. The views and opinions of authors expressed herein do not necessarily state or reflect those of the United States Government or any agency thereof. 


\section{DISCLAIMER}

Portions of this document may be illegible in electronic image products. Images are produced from the best available original document. 


\title{
Modification of the Current Profile in DIII-D by Off-Axis Electron Cyclotron Current Drive
}

\author{
T.C. Luce, Y.R. Lin-Liu, R.W. Harvey, ${ }^{1}$ G. Giruzzi, ${ }^{2}$ J.M. Lohr, \\ C.C. Petty, P.A. Politzer, R. Prater, and B.W. Rice 3
}

General Atomics, P.O. Box 85608, San Diego, California 92186-5698

${ }^{1}$ CompX, 12839 Via Grimaldi, Del Mar, California 92014

${ }^{2}$ CEA, Centre d'Études de Cadarache, F-13108 Saint Paul lez Durance, France

${ }^{3}$ Lawrence Livermore National Laboratory, Livermore, California 94551-9900

\begin{abstract}
Localized non-inductive currents due to electron cyclotron wave absorption have been measured on the DIII-D tokamak. Clear evidence of the non-inductive currents is seen on the internal magnetic field measurements by motional Stark effect spectroscopy. The magnitude and location of the non-inductive current is evaluated by comparing the total and Ohmic current profiles of discharges with and without electron cyclotron wave power. The measured current agrees with Fokker-Planck calculations near the magnetic axis, but exceeds the predicted value as the location of the current drive is moved to the half radius.
\end{abstract}

\section{Introduction}

Control and sustainment of the current profile by non-inductive means is key element of the DIII-D tokamak research program. To realize a practical advanced tokamak scenario, it appears necessary to maintain the current profiles which are created by transient means in order to sustain the high levels of fusion performance. In the absence of any demonstrated pressure profile control to manipulate the bootstrap current, direct current drive is required. Non-inductive current drive by electron cyclotron waves (ECCD) is the technique planned for advanced tokamak demonstration on DIII-D. ECCD has several advantages for DIII-D which carry forward to a future fusion power plant. The location and localization of the current drive is determined by the illumination of the cyclotron resonance by the launched waves. The location can be controlled almost independently of the plasma parameters by 
steering of the launched waves by quasi-optical means or in conjunction with changes in the plasma by changing the total magnetic field. Because the waves are small $(1-3 \mathrm{~mm})$ compared to the launching structures, the illumination of the resonance can be made quite small, down to the intrinsic absorption length under ideal conditions. Because the waves propagate in vacuum and can be transmitted to the fusion device at high power density $(\sim 1 \mathrm{GW} / \mathrm{m} 2$ achieved in vacuum transmission line), substantial power can be applied to a tokamak through a small aperture without material structures near the plasma. The experiments reported here represent a proof-of-principle demonstration that localized noninductive current driven by electron cyclotron waves can be obtained in a tokamak.

\section{Experimental Conditions}

For the experiments reported here, two gyrotron oscillators operating at $110 \mathrm{GHz}$ were employed. The range of power delivered to the plasma was $0.95-1.14 \mathrm{MW}$ for generated powers of 1.23-1.43 MW, giving a typical transmission efficiency of $75 \%-80 \%$. Most of the non-transmitted power is lost in conversion of the non-Gaussian output from the gyrotron to a Gaussian beam suitable for coupling to waveguide. The power is transmitted to the tokamak by evacuated transmission line which contains a pair of grooved miter bends which can be oriented to polarize the beam to nearly any linear or elliptical polarization. The beam is launched into the tokamak using a pair of turning mirrors. For these current drive experiments, the final mirror has a toroidal facet angle of $19^{\circ}$ to direct the beam in the direction to enhance the existing current for the standard operation of DIII-D. The poloidal aiming of the beam can be changed between tokamak pulses. The EC waves at $110 \mathrm{GHz}$ are absorbed in DIII-D near the second harmonic of the electron cyclotron frequency. The polarization corresponding to $\mathrm{X}$ mode dispersion relation (for $k_{\|}=0, \vec{k} \perp \vec{B} \perp \vec{E}$ ) is launched since it is strongly absorbed for the DIII-D conditions.

Discharges without MHD instabilities are needed to successfully apply the current drive analysis technique described below. To avoid sawteeth, a neutral beam is injected into the plasma during the current ramp to raise the temperature as shown in Fig. 1. This slows the resistive evolution of the current profile in order to delay the appearance of a $q=1$ surface and sawteeth. To avoid an $\mathrm{H}$-mode and ELMs, plasmas are biased upward with the grad-B drift downward. Periods of non-diffusive evolution of the poloidal flux are observed, and are correlated with bursts of low- $n$ MHD at very low amplitude. These seem to be related to the minimum $q$ reaching a low-order rational value. These modes rapidly disappear after the crossing through the rational surfaces. Because the resistive evolution is slowed by the NB heating, sufficient time is available between such events to analyze the current drive with good accuracy. 


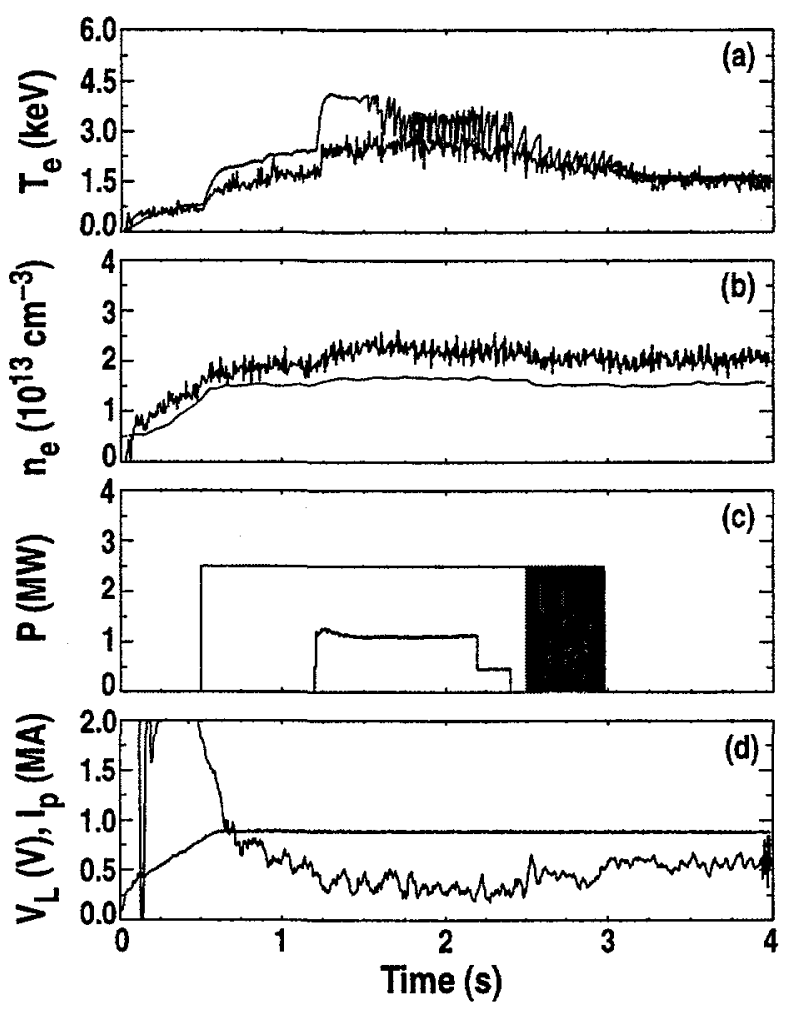

Fig. 1. Time history of a typical ECCD discharge. (a) Electron temperature from central ECE (upper trace) and Thomson scattering near $\rho=0.25$. (b) Electron density from Thomson scattering near $\rho=0.25$ (upper trace) and line-averaged $\mathrm{CO}_{2}$ laser interferometry. (c) Neutral beam power (upper trace) and EC power (arbitrary units). (d) Loop voltage and plasma current.

\section{Current Drive Analysis}

The non-inductive current density is inferred from time sequences of magnetic equilibrium reconstructions [1]. The interior magnetic fields are measured directly by motional Stark effect (MSE) spectroscopy of the deuterium atoms injected by the same neutral beam used to slow the resistive evolution [2]. The MSE data and the magnetics signals are used as constraints on solutions to the Grad-Shafranov equation. The result is a map of poloidal flux $\psi$ as a function of space and time. Spatial derivatives of $\psi$ give the current density parallel to the magnetic field $\left(J_{\|}\right)$as function of $\rho$, the square root of the toroidal flux normalized to its edge value. The time derivative of $\psi$ at fixed $\rho$ gives the electric field parallel to the magnetic field $\left(E_{\|}\right)$. Assuming neoclassical conductivity ( $\sigma_{\text {neo }}$ ) (validated in Ref. 1 ), the total non-inductive current density is given by $J_{\mathrm{NI}} \equiv J_{\|}-\sigma_{\text {neo }} E_{\|}$. This includes bootstrap current, neutral beam current, and electron cyclotron current. To isolate the effect of ECCD, the results are compared to those from a discharge with identical shape, current, and toroidal field with only neutral beam and bootstrap current. 
The difference in $J_{\mathrm{NI}}$ for these two cases is attributed to ECCD. The small differences in electron density $(n)$, electron temperature $\left(T_{\mathrm{e}}\right)$, or impurity content $\left(Z_{\text {eff }}\right)$ are taken into account by adjusting the $J_{\mathrm{NI}}$ in the fiducial discharge by the theoretical scaling. Note that the absolute value and distribution of theoretical non-inductive current is not used in the correction; only the ratio is applied. This correction is usually a small fraction of the $J_{\mathrm{NI}}$ for the fiducial.

\section{ECCD Near the Magnetic Axis}

Application of the above technique to discharges with the intersection of the second harmonic resonance and the EC beam near $\rho=0.15$ yields clear evidence of localized ECCD. The four steps of the analysis are shown in Fig. 2. The total $J_{\| l}$ is shown in Fig. 2(a) for both the ECCD and neutral beam only case. A clear modification of the current density is observed both at the predicted resonance location in $\rho$ and interior to this. This modification is apparent in the magnetic field pitch angles measured by MSE as shown in Fig. 3. The pitch angle is simply the angle whose tangent is the ratio of the poloidal to the toroidal field. The greater separation between the signals at $\rho=0.1$ and $\rho=0.25$ for the ECCD case indicates that the current enclosed between these flux surfaces has increased and is localized to this location.
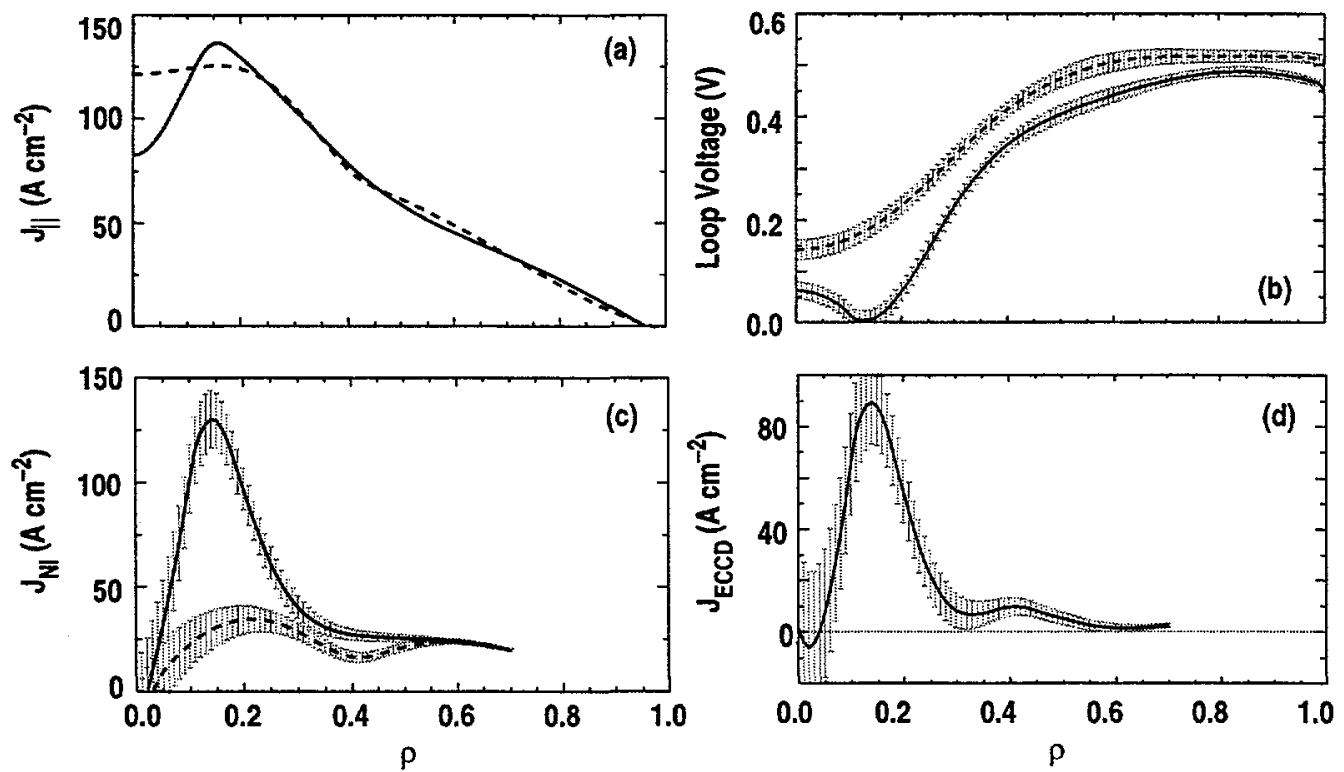

Fig. 2. Analysis of the non-inductive current drive for the case near the magnetic axis. Plasma parameters are $B=1.76 \mathrm{~T}, I=0.89 \mathrm{MA}, \bar{n}=1.8 \times 10^{19} \mathrm{~m}^{-3}, P_{\mathrm{EC}}=1.1 \mathrm{MW}$. (a) Total parallel current density, (b) loop voltage, (c) non-inductive current density, (d) electron cyclotron current density. In (a)-(c), the solid lines are the ECCD case and the dashed lines are the NB fiducial. The analysis is for $t=1.3 \mathrm{~s}$ using data from $t=1.2-1.4 \mathrm{~s}$. 


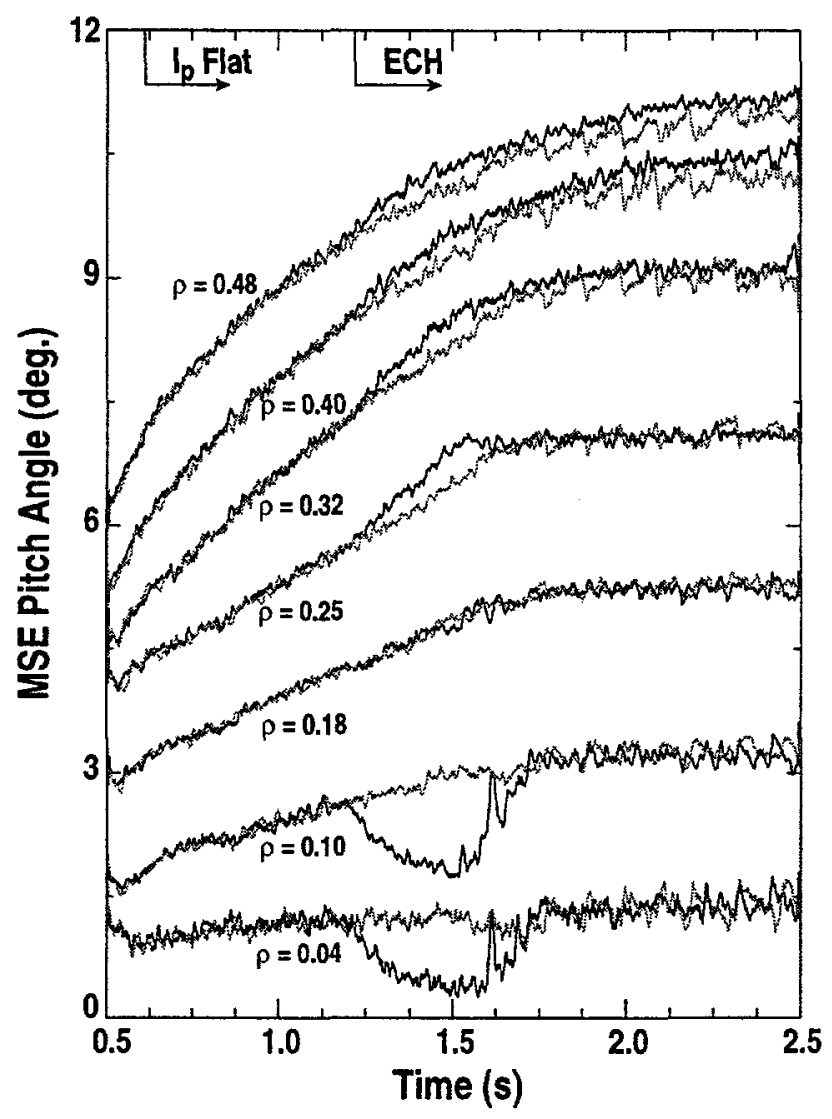

Fig. 3. MSE pitch angle measurements versus time for the ECCD case (heavy line) shown in Fig. 2 and its NB fiducial (light line). Data are smoothed over $10 \mathrm{~ms}$ as used in the analysis.

It may be somewhat surprising that the current density in the center can change so rapidly in a hot plasma. A resistive evolution simulation for this discharge, shown in Fig. 4, indicates that a drop in the central current density and a rise at the resonance location are easily observed even at $0.1 \mathrm{~s}$ into the EC pulse. The simulation uses fixed geometry and kinetic profiles, and steps forward the neoclassical Ohm's law with the addition of a current source due to ECCD calculated from ray tracing and linear kinetic theory. The initial condition for the current density profile is taken to be the measurement at the time the EC power is turned on. The kinetic profiles are fixed to be those at the end of the ECCD analysis since this is the time of highest conductivity and therefore would impose an upper limit on the resistive evolution time scale. The simulation shows that the changes in $J_{\|}$seen in Fig. 2(a) are consistent with resistive evolution.

The change in the MSE pitch angles seen in Fig. 3 is, in principle, consistent with the generation of a radial electric field. However, the possibility that the EC waves generate a radial electric field rather than current drive can be ruled out. The first piece of evidence is the consistency with the neoclassical Ohm's law shown in Fig. 4. Furthermore, it is 


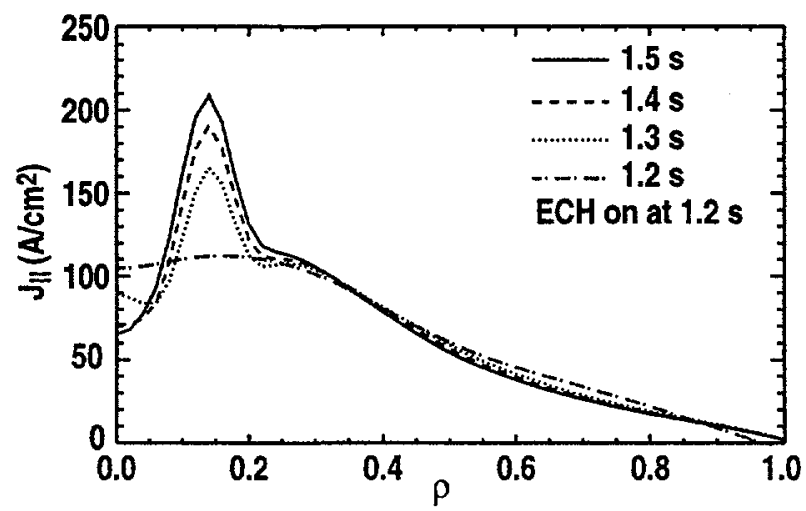

Fig. 4. Results of a resistive diffusion simulation of the effects of ECCD near the magnetic axis. The $J_{\|}$at and interior to the resonance location change noticeably in the first $0.1 \mathrm{~s}$.

possible to calculate the change in toroidal rotation necessary to generate the radial electric field which changes the MSE pitch angles by an amount to mimic the current drive. The observed change in the MSE pitch angles would require an order of magnitude increase in toroidal rotation while the measured toroidal rotation is unchanged or drops slightly. All of the direct measurements and consistency checks indicate the change in the MSE signals is a change in current density due to ECCD.

The time behavior of $\psi$ at fixed $\rho$ gives the $E_{\|}$across the radius or equivalently the loop voltage ( $\left.V_{\mathrm{L}}=2 \pi R E_{\|}\right)$shown in Fig. $2(\mathrm{~b})$. The loop voltage is a true flux function. The error bars shown in Fig. 2(b) are the uncertainty in finding the slope of a straight line through the time sequence of equilibria at constant $\rho$ to determine $\partial \psi / \partial t$. Typically, equilibria are generated at time slices $10 \mathrm{~ms}$ apart using $10 \mathrm{~ms}$ time averaging of the input data. The loop voltage is calculated every $10 \mathrm{~ms}$ using a fit over typically $\pm 100 \mathrm{~ms}$ around the time of interest. Shorter samples yield larger error bars, while longer samples can run into the problem that a straight line does not describe well the time history. The uncertainty on the loop voltage dominates the uncertainty on $J_{\mathrm{NI}}$, and the error bars in subsequent figures are the uncertainty in the loop voltage propagated through the analysis. Note that in neither the ECCD nor the neutral beam case is the loop voltage fully relaxed to its equilibrium value. This means that the standard technique of determining the non-inductive current by measuring the edge loop voltage and assuming the voltage is constant across the radius would fail to accurately determine the driven current in these cases.

The non-inductive current density clearly peaks slightly off-axis, as shown in Fig. 2(c). Taking the difference with the neutral beam discharge shows a peaked ECCD profile at the resonance location [Fig. 2(d)]. The peak value is $>2$ standard deviations $(2 \sigma)$ from zero, and there is more than $1 \sigma$ from the peak to the half maximum. The total current out to $\rho=0.3$ is $92 \pm 29 \mathrm{kA}$, where the uncertainty in the current is the integral of the area 
between the top of the error bars and the measured curve. This is a conservative estimate of the uncertainty in the current. The large uncertainty in evaluating the total current is not indicative of uncertainty that there is current drive as is evident by observing the MSE data in Fig. 3.

The current $(97 \mathrm{kA})$ predicted by a quasilinear Fokker-Planck calculation [3] using the measured kinetic parameters and the inferred $E_{\|}$is in excellent agreement with the measured value; however, the calculated profile is narrower than that measured in the experiment. At present, it is not possible to attribute the broadening in the measurement to the finite resolution of the analysis technique, broadening of the launched beam by some unknown effect, or transport of the fast electrons carrying the current. Efforts to assess the resolution of the analysis technique are underway.

\section{ECCD Near the Half Radius}

Clear evidence of ECCD near the half radius is found using the same analysis technique applied above. Figure 5(a)-(d) shows the same steps of the analysis described above. The modification of $J_{\|}$is much less evident because the local ECCD is less than the existing total
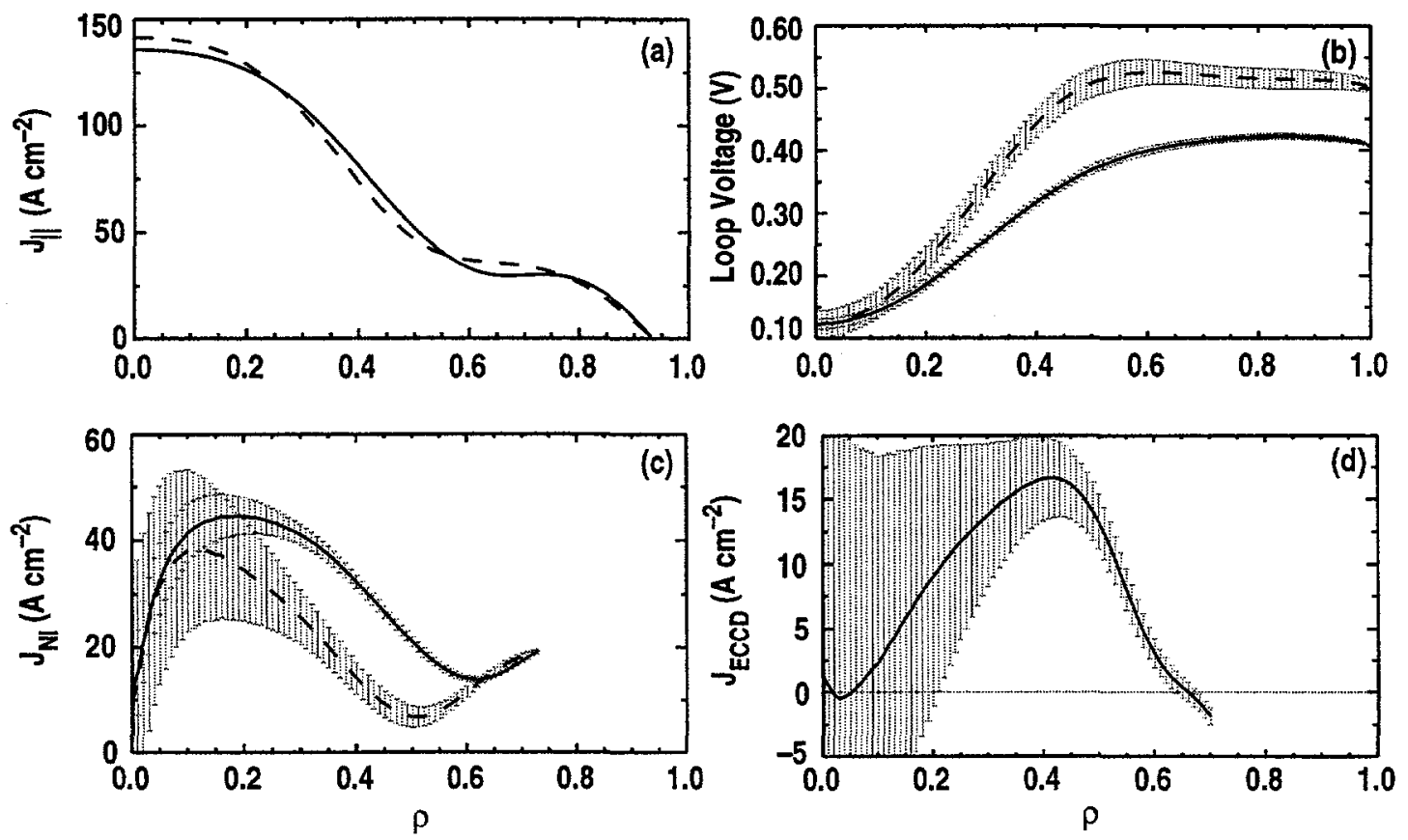

Fig. 5. Analysis of the non-inductive current drive for the case near the half radius. The plasma parameters are $B=1.66 \mathrm{~T}, I=0.84 \mathrm{MA}, \bar{n}=1.8 \times 10^{19} \mathrm{~m}^{-3}, P_{\mathrm{EC}}=0.99 \mathrm{MW}$. (a) Total parallel current density, (b) loop voltage, (c) non-inductive current density, (d) electron cyclotron current density. In (a)-(c), the solid lines are the ECCD case and the dashed lines are the NB fiducial. The analysis is for $1.54 \mathrm{~s}$ using data from $t=1.44-1.64 \mathrm{~s}$. 
$J_{\|}$, and any modification comes from the resistive diffusion. The main effect is that significantly less loop voltage is required to sustain the current profile [Fig. 5(b)].

The reduction in loop voltage can be seen directly in the MSE signals shown in Fig. 6. A rough estimate of $E_{\|}$is given by the toroidal component of Faraday's law:

$$
E_{\phi}=\frac{1}{R} \int d R^{\prime} R^{\prime} \frac{\partial B_{z}}{\partial t}
$$

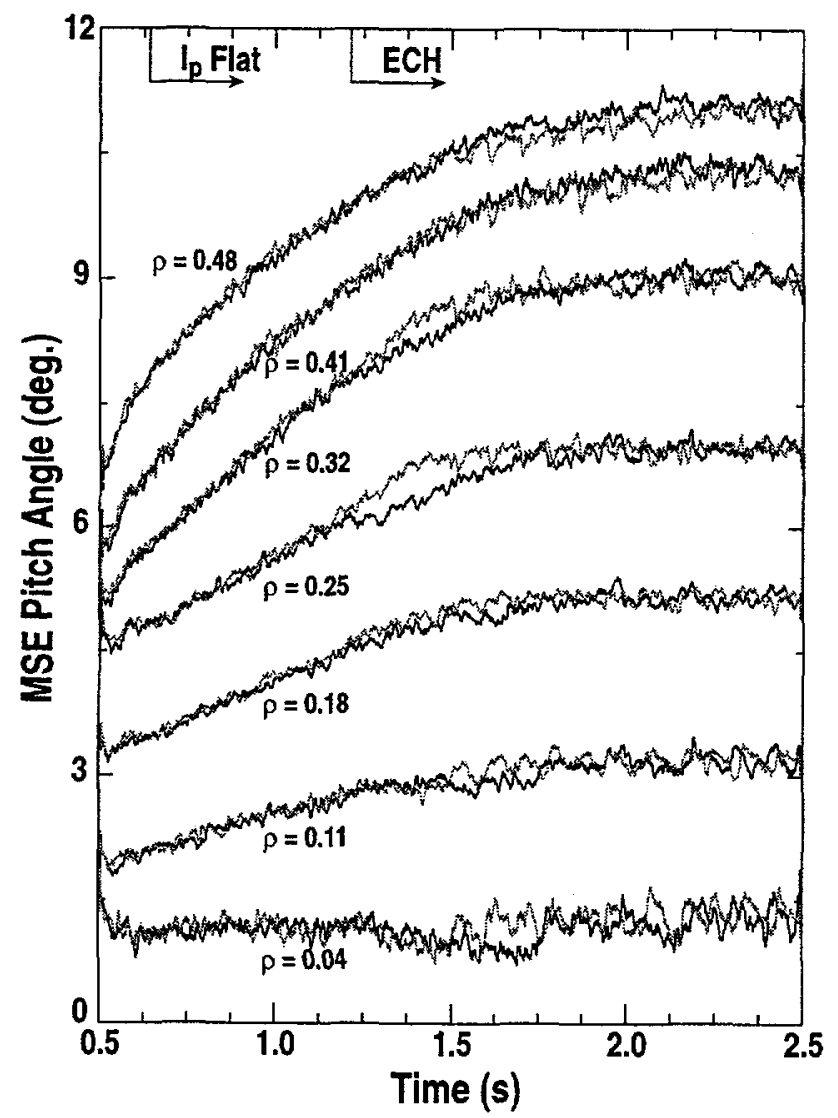

Fig. 6. MSE pitch angle measurements versus time for the ECCD case shown in Fig. 5 (heavy line) and its NB fiducial (light line). Data are smoothed over $10 \mathrm{~ms}$ as used in the analysis.

Assuming all of the change in the pitch angle is due to $B_{z}$, the reduction in the change in pitch angle indicates a smaller loop voltage on the ECCD case starting at $\rho \simeq 0.25$. The calculation of $J_{\mathrm{NI}}$ [Fig. 5(c)] shows that this is due to a local addition of non-inductive current at exactly the location where ECCD is expected. This is clearly shown in Fig. 5(d) where the $J_{\mathrm{EC}}$ from the analysis is shown. Again, the error bars indicate a well-resolved peak to the outside, but substantial uncertainty toward the center. As in the case with ECCD near the magnetic axis, the measured current profile is substantially broader than the 
predicted profile. However, in this case the total current is also substantially larger, $92 \pm$ $39 \mathrm{kA}$ compared with $39 \mathrm{kA}$ calculated. The larger driven current in the analysis cannot be due to a finite resolution effect since the analysis technique accounts for all of the applied poloidal flux by design. Any finite resolution effects can only redistribute current, not increase it.

\section{Comparison to Theory}

A detailed comparison to theory is presented in a separate publication [4] and only the results will be summarized here. The results presented in the previous two sections are typical of the entire ECCD experimental database. For cases with the resonance location at $\rho=0.1-0.3$, the measured current using the analysis technique described here and the predicted current using the quasilinear Fokker-Planck code are in very good agreement. For cases with $\rho=0.3-0.5$, the measured current is substantially higher than the prediction. In all cases, the measured profile is broader than the calculated profile. (The Fokker-Planck code employs a full ray-tracing calculation with multiple rays to model the refraction and finite-beam divergence effects.) Since the known temperature and density dependences are included in the calculation, it is postulated that the discrepancy is due to a simplified treatment of the trapped particle effects in the theoretical calculation. The effect of trapped particles is a subtractive effect (the "Ohkawa effect" [5]), so the impact of lessening the influence of trapped particles would be to add an offset to the theoretical predictions rather than a constant multiplier. Calculations to assess the current drive expected in the absence of trapped particles have been performed with the Fokker-Planck code simply by eliminating the magnetic well. The predicted current is in all cases much higher than the measured value, indicating that a reduction in the effective trapped electron fraction can explain the measurements. The leading candidate for a physical model is the inclusion of finite collisionality in the theoretical treatment. The standard Fokker-Planck treatment with a bounce-averaged collision operator does have a finite collision operator in all of velocity space, but imposes a strict boundary condition between trapped and passing particles down to zero velocity. Clearly, this is not strictly true, and finite collisionality corrections could be significant since the first-order corrections are expected to scale with $\left(v_{*}\right)^{1 / 2}$. The first analytic work in this area using an ad hoc connection formula to interpolate a linear adjoint response between the exactly tractable limits of collisionless and infinite connection length is reported at this meeting [6]. The enhancement of the predicted current drive efficiency is quite modest and is not enough to match the measurements. This linear adjoint approach does not include quasilinear and $E_{\|}$effects. A full 3-D Fokker-Planck treatment with 2-D velocity space and 1-D along the magnetic field lines is in progress. 


\section{Summary and Future Plans}

Localized ECCD out to the half radius has been clearly demonstrated in these proof-ofprinciple experiments. The effects indicated in the complete analysis can be directly observed in the MSE pitch angle measurements. The time scales for the observed current profile modifications are consistent with resistive evolution. The experiments indicate that the effect of trapped electrons is substantially less than that predicted by standard theory in the collisionless limit. Work is in progress to provide current drive predictions at arbitrary collisionality.

The near term plans are to begin experiments with an ELMing $\mathrm{H}$-mode to test for possible mode conversion [7] or unexpected refractive effects. These experiments are a prelude to the first current profile control and tearing mode stabilization experiments. A system composed of four gyrotrons is planned for 2000, increasing to six gyrotrons in 2001.

\section{Acknowledgment}

This work was supported by the U.S. Department of Energy under Contracts DEAC03-99ER54463 and W-7405-ENG-48.

\section{References}

[1] C.B. Forest et al., Phys. Rev. Lett. 73, 2244 (1994).

[2] B.W. Rice et al., Phys Rev. Lett. 79, 2394 (1997).

[3] R.W. Harvey and M.C. McCoy, "The CQL3D Fokker-Planck Code," in Advances in Simulation and Modeling of Thermonuclear Plasmas (Proc. IAEA Technical Committee Meeting, Montreal, 1992), IAEA, Vienna (1993), p. 498.

[4] T.C. Luce et al., "Generation of Localized Non-Inductive Current by Electron Cyclotron Waves on the DIII-D Tokamak," General Atomics Report GA-A23018 (1999), submitted to Physical Review Letters.

[5] T. Ohkawa, "Steady-State Operation of Tokamaks by r-f Heating," General Atomics Report GA-A13847 (1976).

[6] Y.R. Lin-Liu et al., "Modeling of Trapped Electron Effects on Electron Cyclotron Current Drive for Recent DIII-D Experiments," this conference.

[7] R. Dumont and G. Giruzzi, Phys. Plasmas 6, 660 (1999). 